\title{
Tuberous Sclerosis Complex Associated with Autism Spectrum Features and Bumetanide as a Pharmacological Indication: A Case Report
}

\author{
Maria Cristina Brió ${ }^{*}$, Mariana Fazzina ${ }^{1 \#,}$ Melina Chindi ${ }^{2 \dagger}$ \\ ${ }^{1}$ Independent Author, Buenos Aires, Argentina \\ ${ }^{2}$ Independent Author, Bahia Blanca, Argentina \\ Email: *mariacristinabrio@gmail.com
}

How to cite this paper: Brió, M.C., Fazzina, M. and Chindi, M. (2021) Tuberous Sclerosis Complex Associated with Autism Spectrum Features and Bumetanide as a Pharmacological Indication: A Case Report. Open Journal of Psychiatry, 11, 202-213.

https://oi.org/10.4236/ojpsych.2021.113016

Received: May 1, 2021

Accepted: July 19, 2021

Published: July 22, 2021

Copyright $\odot 2021$ by author(s) and Scientific Research Publishing Inc. This work is licensed under the Creative Commons Attribution International License (CC BY 4.0).

http://creativecommons.org/licenses/by/4.0/

\begin{abstract}
A wide variety of genetic and non-genetic pathologies share serious psychiatric symptoms, which determine a poor quality of life for patients and their families. To evaluate whether bumetanide, a drug initially developed as a diuretic and currently analyzed for a new indication in patients with severe neuropsychiatric pathologies, could improve the disruptive and self-injurious behaviors secondary to Tuberous Sclerosis Complex (TSC) and characteristic of the autistic spectrum the case of this 6-year-old patient is considered. Following preclinical and clinical evidence of the efficacy of bumetanide in $\mathrm{Tu}$ berous Sclerosis and other neurodevelopmental disorders, the drug may alleviate the psychiatric manifestations (TAND) of Tuberous Sclerosis pathology. This would allow avoiding the excessive prescription of antipsychotic drugs indicated to control disruptive behaviors. Methodology: The Administración Nacional de Medicamentos, Alimentos y Tecnología Médica (ANMAT) approval was requested for compassionate use since it is not an approved drug in Argentina for this indication. The patient was evaluated with laboratory tests at T0, T1, T2, T3 and T4, corresponding to the basal moments, of 7 days, 30 days, 60 days and 90 days after starting the medication. Likewise, behavior was evaluated with the Aberrant Behavior Checklist (ABC) at the same times described. CARS was used for autistic characteristics and TAND Checklist for psychiatric disorders, both at the beginning. Bumetanide was administered at $1 \mathrm{mg} /$ day and increased to $1.5 \mathrm{mg} /$ day one month after the first dose. Results:
\end{abstract}

\footnotetext{
${ }^{\star}$ Children and Adolescent Psychiatrist. CSM N1. DGSAM. Master's Degree in Psychoneuropharmacology, U. Favaloro. Master's Degree in Statistics, Universidad de Buenos Aires. Corresponding author.

\#Clinical Psychiatrist. Master's Degree in Pharmacology, Universidad de Buenos Aires.

${ }^{\dagger}$ Occupational Therapist. Irel (Instituto de Rehabilitacion Integral), Bahia Blanca, Argentina.
} 
We observed, in this case, the primary endpoint, irritability, showed moderate improvement. On the contrary, hyperactivity, attention, sociability and better connection through gaze experienced and evident improvement. Regarding isolation scales and stereotyped behaviors, an important improvement was found after 3 months of treatment with bumetanide, an antagonist NKCC1, evaluated through the Aberrant Behavior Checklist (ABC). On the other hand, no remarkable adverse effects were observed.

\section{Keywords}

Bumetanide, Tuberous Sclerosis Complex, Autism Spectrum Disorder, Antagonist NKCC1

\section{Introduction}

A wide variety of genetic and non-genetic pathologies share serious psychiatric symptoms, which determine a poor quality of life for patients and their families.

The search for new molecules that improve certain psychiatric characteristics associated with the underlying pathology is intense but not always fruitful.

To evaluate whether bumetanide, a drug initially developed as a diuretic and currently analyzed for a new indication in patients with severe neuropsychiatric pathologies, could improve the disruptive and self-injurious behaviors secondary to Tuberous Sclerosis Complex (TSC) and characteristic of the autistic spectrum the case of this 6-year-old patient is considered.

The pathology named Tuberous Sclerosis Complex (TSC) is an autosomal dominant disease caused by mutations in the genes encoding TCS1 (Hamartin) and TSC2 (Tuberin). The TSC1 and TSC2 proteins are required for the suppression of the activity of the target of rapamycin, mTOR. Mutations that can occur in either of the two ET genes, TSC1 (hamartin) or TSC2 (tuberin) are found in over $85 \%$ of ET patients. The proteins encoded by these two genes form a tumor suppressor complex acting through a Ras homologous pathway enriched in a brain protein (Rheb) limiting the activation of mTOR (mTORC1). When TSC1 or TSC2 are deficient, $\mathrm{mTORC} 1$ is constitutively overexpressed, which will result in abnormally high cell growth, proliferation and protein synthesis [1] [2].

Spontaneous genetic mutations occur in $2 / 3$ cases. The incidence of TSC is about 1 in 6000 live births. It presents with a wide range of manifestations caused by localized cellular overgrowth leading to benign tumors (hamartomas) in multiple organs [3]. Because of brain involvement, Tuberous Sclerosis Complex is associated with a wide range of psychiatric and neurodevelopmental disorders, Epilepsy is estimated to occur in $72 \%$ to $85 \%$ of patients, generally with insufficient response to pharmacological treatment. On the other hand, psychiatric disorders (TAND) comprise a set of affective, behavioral, and developmental symptoms that affect $90 \%$ of patients. The most frequently observed are within the autistic spectrum disorder [4] [5]. 
One of the latest treatments proposed for Tuberous Sclerosis is one that targets chloride homeostasis. Several studies implicated altered neuronal chloride levels between and in tubers. More specifically, altered ratios between the NKCC1 cotransporter and the KCC2 cotransporter may affect GABA polarity and cause unwanted depolarization (elevated NKCC1/KCC2). Bumetanide, an antagonist of selective NKCC1 transporters, may act on GABAergic neurotransmission, otherwise implicated in autism spectrum pathologies [6] [7].

The drug regulates neuronal chloride concentration by inhibiting the NKCC1 cotransporter and consequently reinstating GABAergic hyperpolarization currents [8].

Various investigations in animal models of autism showed that drugs that impact on GABAergic neurotransmission can attenuate symptoms linked to the pathology [9].

Following preclinical and clinical evidence of the efficacy of bumetanide in Tuberous Sclerosis and other neurodevelopmental disorders, bumetanide can alleviate the psychiatric manifestations (TAND) of Tuberous Sclerosis pathology.

It is important to point out the difficulties in initiating treatment with this drug, which is new for this indication, since the drug was not available in Argentina. In order to use it in Argentina, it was necessary to request ANMAT approval for compassionate use and import it, since the drug is not available in the country. This program was approved by the European Medicines Agency (EMA) based on the safety data known for its wide use as a diuretic. In summary, the safety of the drug is the same as for its use as a diuretic and is the same as that supported by the clinical studies currently underway. The option of participating in the clinical studies as a research site and thus obtaining the drug was evaluated but it was not possible to enter with the study in progress. We had the opportunity to establish communication with the Principal Investigator and his co-investigator, who provided us with information about the drug, the dosage applied in the study and the status of the study (Lemonnier E, 2020, personal communication).

Currently, studies investigating the efficacy of bumetanide on the central symptoms of autism are still in phase 3 in 40 centers across Europe, Brazil and USA. Patient recruitment is slow, since the indication is very narrow. It is assumed that approval for the indication in autism will be by the end of 2023, if the results are favorable (Ben-Ari Y, 2021, personal communication).

The indication should be: Treatment of Autism Spectrum Disorder (ASD), more specifically Treatment of the core symptoms of Autism Spectrum Disorder (ASD) in children and adolescents aged 2 - 18 years.

Authorization is granted to study a subset(s) of the pediatric population affected by the developing pediatric population aged 2 to less than 18 years, using the age-appropriate oral liquid formulation (Ben-Ari Y, personal communication). 


\section{Case Presentation}

\subsection{Reason for Consultation}

Disruptive behaviors, crying, irritability, self-injury with evidence of bite injuries, severe sleep disturbances and increased appetite in the context of tuberous sclerosis.

\subsection{Personal History}

M. was diagnosed at 3 months of age with Tuberous Sclerosis Complex.

Additionally, at 5 months, West syndrome was diagnosed, and she was treated with different pharmacological schemes with an inadequate electro-clinical evolution and persistence of crises.

At 2 years of age, cannabidiol oil (Charlotte, USA) is incorporated, which reduces the number of daily crises. This treatment is currently suspended. The developmental parameters showed a severe neuromadurative delay. She has no language, no pointing, no sphincter control, a stereotyped behavior, makes herself understood when she needs something. On the other hand, she shows extreme sensory dysregulation that prevents daily activities such as bathing, dressing, undressing, sleeping, and feeding.

At the age of 5 and a half years she began to cry, had sleep problems that worsened with the incorporation of self-injurious behaviors such as compulsive biting in front of any stimulus that was unpleasant for her. She was prescribed with antipsychotics with little efficacy.

In July 2020, a sensory profile evaluation was performed which showed that the patient presented standard deviations in the areas of seeking, avoidance, sensory and spectator register. At sensory level 60/70 and at behavioral level $83 / 100$. A great deviation is observed in the scale of compulsive, self-injurious and stereotyped behaviors.

Psychiatric symptomatology was evaluated with the TAND Checklist, which showed the behaviors presented throughout the day: mood swings, explosive reactions, self-injury (biting and hitting), poor eye contact, total lack of language, repetitive behaviors, rigidity or inflexibility to changes, difficulty paying attention, restlessness, impulsivity and sleep disorders.

\subsection{History of Pharmacological Treatment}

Cannabis oil (Charlotte, Epifractan 2\%) was prescribed on 3 occasions with poor response and ketogenic diet had to be suspended due to lack of tolerance.

The pharmacological approach she currently receives: Vigabatrin $2000 \mathrm{mg} / \mathrm{day}$, Divalproate sodium $375 \mathrm{mg} /$ day, lacosamide $50 \mathrm{mg} /$ day, Enalapril $10 \mathrm{mg} /$ day, Barex 1/2 sachet/day, Esomepromazol $20 \mathrm{mg} /$ day, Quetiapine $150 \mathrm{mg} /$ day, Levomepromazine $16 \mathrm{mg} /$ day, Risperidone decreasing. N-acetyl cysteine $1200 \mathrm{mg} /$ day and bumetanide $1 \mathrm{mg} /$ day are incorporated. One month after starting the dose of $1 \mathrm{mg} / \mathrm{d}$ of bumetanide was increased to $1.5 \mathrm{mg} /$ day. 


\section{Methods}

The patient was diagnosed with TSC based in genetic and clinical diagnostic criteria. Autism spectrum disorder comorbid features are present too.

Bumetanide was administered at $1 \mathrm{mg}$ /day and increased to $1.5 \mathrm{mg} /$ day one month after the first dose.

The use the concomitant antiepileptic drugs were allowed.

The Quantitative Checklist for Autism in Toddlers (Q-CHAT) was also used, as it presents symptoms within the spectrum.

The result was 61 (mean girls TEA) $54.6 \pm 14.9$; mean control girls $25.8 \pm 7.7$ ).

Aberrant Behavior Checklist (ABC)

Results:

Irritability subscale: 28 extremely high.

Isolation subscale: 25

Stereotyped behavior subscale: 16

Hyperactivity subscale: 27

Inappropriate language subscale: no language development

CARS (Childhood Autism Rating Scale)

Score: 52, cut-off point 36.5 (From 36.5 severe autism, MSE > 34) [10].

TAND Checlkist, which revealed the following behaviors throughout the day: mood swings, explosive reactions, self-injury (biting and hitting), poor eye contact, total lack of language, repetitive behaviors, rigidity or inflexibility in the face of change, difficulty paying attention, restlessness, impulsivity and sleep disturbances.

\section{Sensory Profile}

The patient showed in the sensory profile [11], standard deviations in the search, avoidance, sensitive and spectator register areas. There is a large deviation from the scale of compulsive, self-injurious and stereotyped behaviors.

\section{Blood, Urine and ECG Assessment}

At the treatment beginning, evaluations were performed with the following results: complete blood and urine lab. ECG. Studies within normal limits.

The pharmacological treatment she was receiving until December 2020 included several antiepileptic and antipsychotic drugs: Vigabatrin $2000 \mathrm{mg} / \mathrm{day}$, Divalproate sodium $500 \mathrm{mg} /$ day, Lacosamide $100 \mathrm{mg} /$ day, Quetiapine $150 \mathrm{mg} /$ day, Levomepromazine $16 \mathrm{mg} /$ day, $\mathrm{N}$-acetyl cysteine, $1200 \mathrm{mg} /$ day and bumetanide 1 $\mathrm{mg} / \mathrm{day}$.

$\mathrm{N}$-acetyl cysteine, $1200 \mathrm{mg} /$ day and bumetanide $1 \mathrm{mg}$ /day were added.

On $2 / 1 / 21$ the patient started with the drug Bumetanide, $1 \mathrm{mg} / \mathrm{d}$ in a titration period, which was added to the pharmacological scheme she was already receiving. The purpose of adding bumetanide is to obtain a behavioral improvement related to the high levels of irritability, hyperactivity, and self-injurious behaviors.

Previously, a complete blood and urine laboratory, ECG and consultation with nephrology were performed. 
The Behavior Checklist-Irritability (ABC-I) was considered the primary endpoint.

On January 9, 2021, after 7 days, the analyses were repeated and showed values within normal limits.

This evaluation scheme will be repeated on days 14, 30 and 60 and 90 days.

IQ: not evaluable, mental age between 1.5 - 2.5 years is presumed.

Figure 1 shows that isolation and hyperactivity are the characteristics that evolved best with medication. Irritability decreased in quantity and intensity but had an oscillating improvement profile. Language was unchanged and sleep improved with sleep hygiene measures.

Table 1 Shows the values obtained in the initial evaluation and after 3 months of treatment. Mean and SD values are shown for age $\leq 6$. ABC-2 Mean and SD. $\geq 6$ years old, Values of symtomsevaluated.

Table 1. ABC-2 values comparative table of mean (SD) and 3 month evaluation.

\begin{tabular}{cccc}
\hline & Mean and SD & Cero Month & Three Month \\
\hline Irritability & $13.8(9.8)$ & 32 & 32 \\
Social Withdrawal & $10.2(8)$ & 25 & 18 \\
Stereotipicbehavior & $5,3(4.9)$ & 16 & 18 \\
Hiperactivity & $20.1(11.7)$ & 27 & 24 \\
Speech & No Language & &
\end{tabular}

Aislamiento: Isolation, Estereotipia: Stereotypy; Hiperactividad: Hyperactivity, Irritabilidad: Irritability.

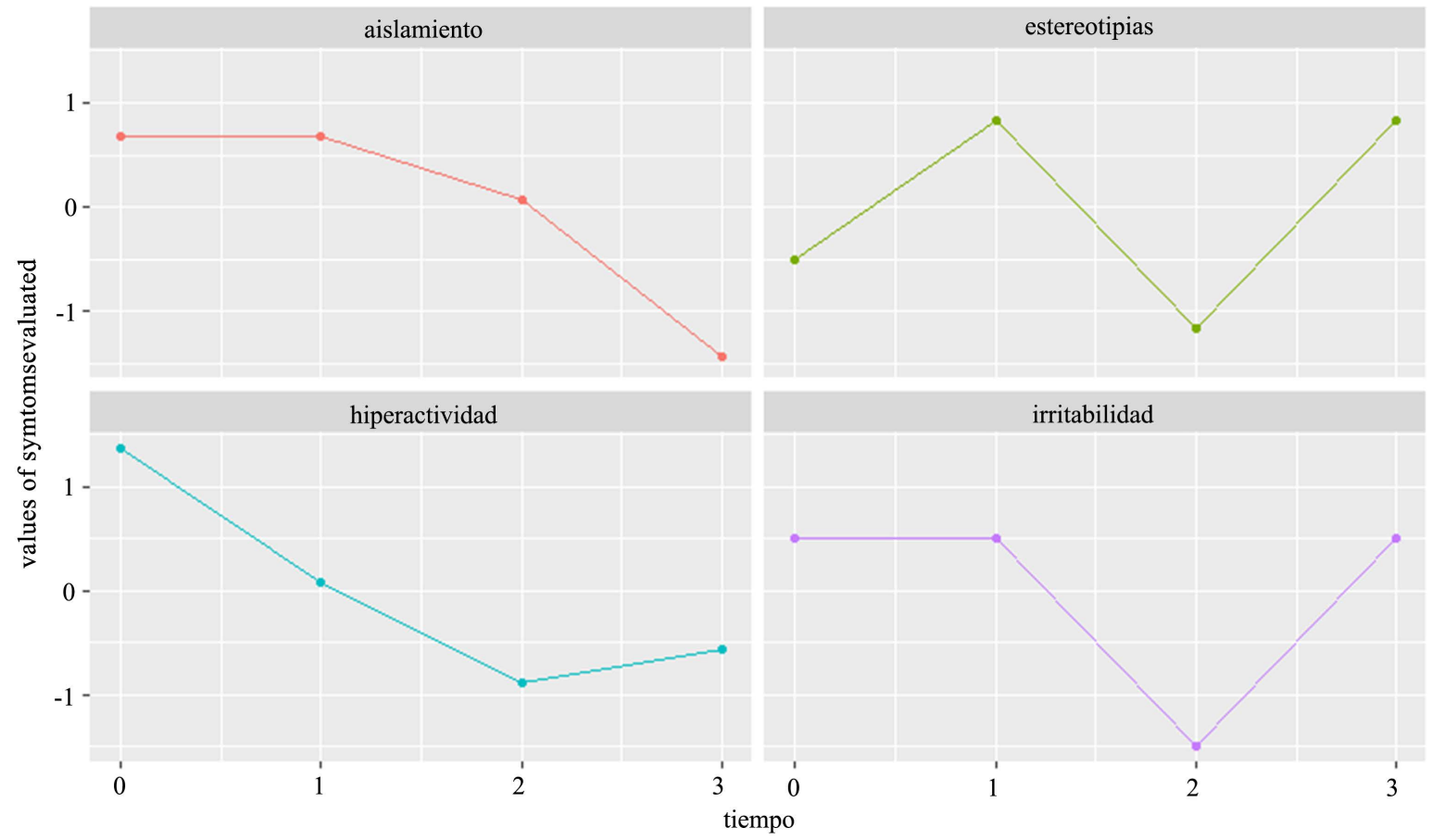

Figure 1. Symptom profile evaluated through ABC-2 in the 3-month period. Aislamiento: Isolation, Estereotipia: Stereotypy; Hiperactividad: Hyperactivity, Irritabilidad: Irritability. 
Table 2 shows the variations of blood parameters over the time analyzed.

With respect to laboratory tests (Figure 2), the values were corrected based on the laboratory results. One of the situations that require more control is the possibility of hypokalemia, or dehydration, since this is a diuretic drug, which can be reversed with potassium supplementation, stabilization of the dose and hydration.

Table 2. Laboratory parameter values in the 3-month period.

\begin{tabular}{ccccc}
\hline & t0 & t1 & t2 & t3 \\
\hline UREA (mg/\%) & 25 & 34 & 29 & 33 \\
CREATININA (mg/\%) & 0.44 & 0.56 & 0.6 & 0.57 \\
TGO (UI) & 18 & 21 & 17 & 16 \\
TGP (UI) & 5 & 5 & 5 & 5 \\
GLUCEMIA (mg/\%) & 77 & 82 & 88 & 85 \\
CALCEMIA (mg/\%) & 9.4 & 9.9 & 10.2 & 10.2 \\
ACIDOURICO (mg/\%) & 3.6 & 5 & 4.7 & 5.5 \\
SODIO (mEq/l) & 140 & 141 & 140 & 139 \\
\hline
\end{tabular}

Ácido Úrico: Uric acid, Glucemia: Glucemia, Creatinina: Creatinine; Urea: Urea, TGO: AST, TGP: ALT, Potasio: Potassium, Sodio: Sodium.

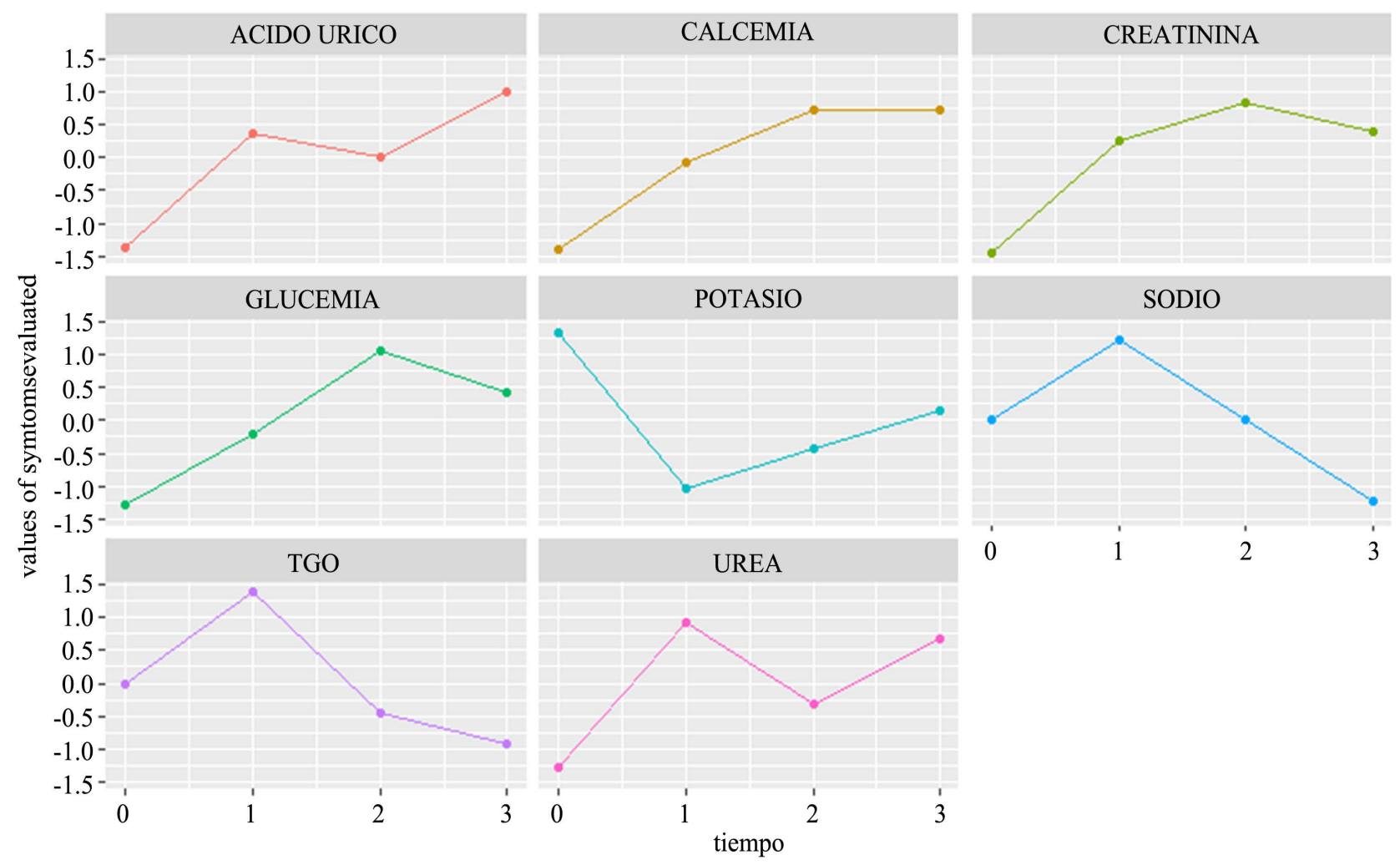

Figure 2. Profile of laboratory parameter values in the 3-month period. Ácido Úrico: Uric acid, Glucemia: Glucemia, Creatinina: Creatinine; Urea: Urea, TGO: AST, TGP: ALT, Potasio: Potassium, Sodio: Sodium. 


\section{Results}

The results observed in this girl showed an improvement in attention, social behavior, hyperactivity and better connection through gaze.

Irritability decreased in number and extent of disruptive episodes, but some events continued to be very intense. She did not show discomfort due to the diuretic effect of the drug. Her blood pressure remained within normal limits. Hydration was controlled with a suitable plan for this purpose.

She has managed to adapt and recognize the therapists, the space, and the materials. Get feedback through smiles, caresses, screams and flaps (of joy at feeling comfortable) and responds to the initiative (games with balls, bubbles, various objects, multisensory room, etc.).

Irritability decreased in number and extent of disruptive episodes, but some events continued to be very intense.

She remains attentive during the sessions, without constantly registering overflows or crises.

Recognizes and looks for objects used within the doctor's office. Maintains gaze at the interlocutor for brief moments. Fixed gaze with more intensity.

Anticipatory behavior is observed as an indicator of understanding in information specific situations and more intensively tolerant of proprioceptive and tactile.

Andel et al. carried out an investigation with 13 children with TS, aged 8 - 21 years. The treatment was well tolerated, with only adverse events related to the diuretic effect of the drug. Improvements were observed in irritability, social behavior, and hyperactivity.

E. Lemonnier and Y Ben-Ari conducted a randomized controlled trial of bumetanide in autistic children. Bumetanide significantly reduced the Childhood Autism Rating Scale, the Clinical Global Impressions and the Autism Diagnostic Observation Schedule values in the most severe cases. Side effects were restricted to an occasional mild hypokalemia that was treated with supplemental potassium [12] [13].

Hadjikhani et al., demonstrated that the administration of bumetanide normalized amygdala activation produced by the limitation of eye contact. These findings are consistent with what the patient experience [14].

In two studies, Hadjikhani et al., measured biomarkers of social cogni tion with bumetanide during an open-label trial. Ten month of treatment improved emotion recognition and enhanced activation in social and emotional perception in some brain areas [15].

Regarding sensory behaviors, although they are not included as central symptoms of the autism spectrum disorder (DSM5), they are highly prevalent characteristics that determine poor social adjustment. Grandgeorge et al. evaluated a 10-year-old girl with Dunn's Sensory Profile after 18 months of bumetanide treatment. The results showed improvement in a wide range of sensory behaviors such as auditory, vestibular, multisensorial, tactile and oral sensory processing 
[16].

Lingli Zhang et al., in an observation study, evaluated efficacy, safety and possible neuropharmacological mechanisms of the bumetanide in young children during 3 months. 83 patients were randomized to bumetanide group $(0.5 \mathrm{mg}$ twice daily) and no bumetanide group. Compared with the control group, the patients who received bumetanide showed a reduction in the severity of the symptoms indicated by the total CARS score and the number of items assigned to a score greater than or equal to 3 [17].

\section{Discussion}

Tuberous Sclerosis Complex (TSC) is a genetic, multisystem disorder with variable phenotypes. It is characterized by autosomal dominant mutations in the TSC1 or TSC2 genes coding for the hamartin protein on chromosome 9q34 and tuberin on chromosome 16q13 respectively, leading to overactivation of the mTOR (target of rapamycin) signaling pathway increasing cell proliferation. Benign tumors represent the typical hallmark of the disease with the central nervous system (CNS), kidney and skin the most frequently affected organs.

Individuals with TSC suffer from a range of psychiatric, intellectual and psychosocial disorders, but assessment and treatment of the behavioral aspects of these patients is infrequent. The most frequently observed psychiatric diagnoses include autism ( $25 \%$ to $50 \%$ ), attention deficit hyperactivity disorder (30\% to $50 \%$ ), as well as depression and anxiety and are associated with variable levels of self- and aggression to others, insomnia and intellectual disability.

Several investigations have identified factors, both organic and environmental, that influence the appearance of aggressiveness in children and adults with genetic disorders linked to intellectual disability. In the case we describe, aggressiveness was a primary endpoint, being this behavioral characteristic responsible for the deterioration of the quality of life of the patient and her family.

Several robust investigations point out to the importance of environmental factors that enable the development and maintenance of aggression through various forms of positive reinforcement of the patient's demands. The role of biological factors and those linked to comorbid syndromes such as Autism Spectrum Disorders (ASD), Attention Deficit Hyperactivity Disorder (ADHD), reduced communication skills and intellectual disability should also not be discounted.

In this girl, the most concerning behaviors were linked to self-injury. Psychiatric symptomatology was assessed with the TAND Checklist, which revealed the following behaviors throughout the day: mood swings, explosive reactions, self-injury (biting and hitting), poor eye contact, total lack of language, repetitive behaviors, rigidity or inflexibility in the face of change, difficulty paying attention, restlessness, impulsivity and sleep disturbances.

Figure 1 shows the improvement in social behavior and hyperactivity. With respect to laboratory tests (Figure 2), the values were corrected based on the la- 
boratory results. One of the situations that require more control is the possibility of hypokalemia, or dehydration, since this is a diuretic drug, which can be reversed with potassium supplementation, stabilization of the dose and hydration. In the other hand, Table 1 shows the variations of blood parameters over the time analyzed.

\section{Conclusions}

The study evaluated whether bumetanide could improve the disruptive and self-injurious behaviors secondary to Tuberous Sclerosis Complex and characteristic of the autistic spectrum the case of this 6-year-old patient was considered.

The beneficial effects of bumetanide, reported in cases of autism, schizophrenia, and other complex comorbid pathologies, are promising, although it requires rigorous investigations that allow more details about its efficacy.

In this particular case, the primary endpoint, irritability, showed moderate improvement. On the contrary, hyperactivity, attention, sociability and better connection through gaze experienced and evident improvement.

Regarding isolation scales and stereotyped behaviors, an important improvement was found after 3 months of treatment with bumetanide, an antagonist NKCC1, evaluated through the Aberrant Behavior Checklist (ABC). On the other hand, no remarkable adverse effects were observed.

Future research is needed for evidence that bumetanide may be a pharmacological agent to treat core autistic spectrum disorders symptoms and complex pathologies associated with them.

Bumetanide, an antagonist of the NKCC1 chlorine $\left(\mathrm{Cl}^{-}\right)$transporter, can reduce the level of intraneuronal $\mathrm{Cl}^{-}$, establishing a balance in the brain excitatory/inhibitory functions, allowing to change the GABAergic activity from excitatory to inhibitory, which would translate into behaviors more adapted to the environment and would result in a better quality of life for the children and their families.

\section{Acknowledgements}

We are grateful to Dr. Y Ben-Ari for helpful comments on the paper and to E. Lemonnier for the comments on their ongoing investigation.

\section{Conflicts of Interest}

The authors have not conflict of interest.

\section{References}

[1] Daniel, E.-F., Mann, L.L., Poryo, M., Graf, N., Von Kries, R., Heinrich, B., Darius, E.F., Marina, F.-B., Ludwing, G., Zemlin, M. and Meyer, S. (2018) Incidence of Tuberous Sclerosis and Age at First Diagnosis: New Data and Emerging Trends from a National, Prospective Surveillance Study. Orphanet Journal of Rare Diseases, 13, Article No. 117. https://doi.org/10.1186/s13023-018-0870-y

[2] Curatolo, P., Moavero, R. and De Vries, P. (2015) Neurological and Neuropsychia- 
tric Aspects of Tuberous Sclerosis Complex. The Lancet Neurology, 14, 733-745.

[3] Davis, P.E., Jurriaan, M.P., Darcy, A.K. and Mustafa, S. (2015) Tuberous Sclerosis: A New Frontier in Targeted Treatment of Autism. Neurotherapeutics, 12, 572-583. https://doi.org/10.1007/s13311-015-0359-5

[4] De Vries, P.J., Whittemore, V.H., Leclezio, L., Byars, A.W., Dunn, D., et al. (2015) Tuberous Sclerosis Associated Neuropsychiatric Disorders (TAND) and TAND Checklist. Pediatric Neurology, 52, 25-35. https://doi.org/10.1016/j.pediatrneurol.2014.10.004

[5] DSM-5 (2013) Manual diagnóstico y estadístico de los trastornos mentales. American Psychiatric Association.

[6] Van Andel, D.M., Sprengers, J.J., Bob, O., Floortie, E.S. and Hilgo, B. (2020) Effects of Bumetanide on Neurodevelopmental Impairments in Patients with Tuberous Sclerosis Complex: An Open-Label Pilot Study. Molecular Autism, 11, Article No. 30. https://doi.org/10.1186/s13229-020-00335-4

[7] Morita, Y., Joseph, H.C., Lauren, R.T., Michelle, I.M., Dwight, D., Chen, Q., Tao, R., Barbara, K.L., Bhaskar, K., Amanda, J.L., Ye, T., Richard, E.S., Daniel, R.W., Joel, E.K. and Thomas, M.H. (2014) Characteristics of the Cation Cotransporter NKCC1 in Human Brain: Alternate Transcripts, Expression in Development, and Potential Relationships to Brain Function and Schizophrenia. Neurobiology of Disease. The Journal of Neuroscience, 34, 4929-4940. https://doi.org/10.1523/JNEUROSCI.1423-13.2014

[8] Shivani, C.K., Kang, S.K. and Shilpa, D.K. (2019) Off-Label Use of Bumetanide for Brain Disorders: An Overview. Frontiers in Neuroscience, 13, Article No. 310. https://doi.org/10.3389/fnins.2019.00310

[9] Mollajani, R., Mohamad, T.J. and Therani-Doost, M. (2019) Bumetanide Therapeutic Effect in Children and Adolescent with Autistic Spectrum Disorder: A Review Study. Basic and Clinical Neuroscience, 10, 433-442.

https://doi.org/10.32598/bcn.9.10.380

[10] Schopler, E., Reichler, R. and Rochen, R. (1988) The Childhood Autism Rating Scale. Western Psychological Services.

[11] Dunn, W. (2014) Perfil Sensorial-2. PsychCorp, Pearson.

[12] Lemonnier, E., Degrez, C., Phelep, M., Tyzio, R., Josse, F., Grandgeorge, M., Hadjikhani, N. and Ben-Ari, Y. (2012) A Randomised Controlled Trial of Bumetanide in the Treatment of Autism in Children. Translational Psychiatry, 2, e202. https://doi.org/10.1038/tp.2012.124

[13] Lemonnier, E., Villeneuve, N., Sonie, S., Serret, S., Rosier, A., Roue, M., Brosset, P., Viellard, M., Bernoux, D., Rondeau, T., Ravel, D. and Ben-Ari, Y. (2017) Effects of Bumetanide on Neurobehavioral Function in Children and Adolescents with Autism Spectrum Disorders. Translational Psychiatry, 7, e1056.

https://doi.org/10.1038/tp.2017.10

[14] Hadjikhani, N., Johnels, J.Å., Lassalle, A., Zürcher, N.R., Hippolyte, L., Gillberg, C., Lemonnier, E. and Ben-Ari, Y. (2018) Bumetanide for Autism: More Eye Contact, Less Amygdala Activation. Scientific Reports, 8, Article No. 3602. https://doi.org/10.1038/s41598-018-21958-x

[15] Hadjikhani, N., Zurcher, N.R., Rogier, O., Ruest, T., Hippolyte, L., Ben-Ari, Y. and Lemonnier, E. (2015) Improving Emotional Face Perception in Autism with Diuretic Bumetanide: A Proof-of-Concept Behavioral and Functional Brain Imaging Pilot Study. Autism, 19, 149-157. https://doi.org/10.1177/1362361313514141

[16] Grandgeorge, M., Eric, L., Céline, D. and Nelle, J. (2015) The Effect of Bumetanide 
Treatment on the Sensory Behaviours of a Young Girl with Asperger Syndrome. BMJ Case Reports, 2014, bcr2013202092. https://doi.org/10.1136/bcr-2013-202092

[17] Zhang, L., Huang, C.-C., Dai, Y., Luo, Q., Ji, Y., Wang, K., Deng, S., Yu, J., Xu, M., Du, X., Tang, Y., Shen, C., Feng, J., Barbara, J.S., Lin, C.-P. and Li, F. (2020) Symptom Improvement in Children with Autism Spectrum Disorder following Bumetanide Administration Is Associated with Decreased GABA/Glutamate Ratios. Translational Psychiatry, 10, Article No. 9. https://doi.org/10.1038/s41398-020-0692-2 